Haben Sie Fragen zur Abrechnung oder zur wirtschaftlichen

\title{
Alt-Unterlagen
}

\section{Beim Ausmisten bitte die Fristen beachten!}

2

Dr. M., Hausarzt-Internistin, Bayern: Wir haben unsere Praxis demnächst für ein paar Tage geschlossen. In dieser Zeit wollten wir uns u. a. von alten Akten befreien. Welche Aufbewahrungsfristen muss ich da beachten?

I MMW-Experte Walbert: Grundsätzlich muss in der Praxis zwischen Unterlagen des Vertragsarztes und Unterlagen des Unternehmers unterschieden werden.

Wenden wir uns zunächst den ärztlichen Aufzeichnungen und Patientenunterlagen zu. Sie unterliegen den berufsrechtlichen Vorschriften und sind mindestens zehn Jahre nach Abschluss der Behandlung aufzubewahren. Hier beginnt der Ablauf der Frist mit dem Jahr, das auf den letzten Eintrag oder Kontakt folgt. Andere gesetzliche Vorschriften können im Einzelnen sogar noch längere Fristen vorschreiben, z. B. bei Strahlentherapie, oder berufsgenossenschaftlicher Behandlung.

In vielen Praxen wird heutzutage nur noch elektronisch dokumentiert. In der Regel ist ein Löschen von Daten hier gar nicht mehr vorgesehen. Die Berufsordnung schreibt aber vor, dass bei der Dokumentation auf elektronischen oder anderen Speichermedien besondere Sicherungs- und Schutzmaßnahmen getroffen werden, um eine Veränderung, Vernichtung oder unrechtmäßige Verwendung zu verhindern.

Sollten in der Praxis noch Papierdokumente aufbewahrt werden, gelten hierfür teilweise kürzere Aufbewahrungsvorschriften (Tab. 1).

Kommen wir nun zu den geschäftlichen Dokumenten. Als Unternehmer sind zwei Fristen von Bedeutung. Eine 6-jährige Aufbewahrungsfrist gilt für Handels- und Geschäftsbriefe, und zwar sowohl für die empfangenen als auch für die Kopien der versandten Unterlagen, insbeson-
Tab. 1 Aufbewahrungsfristen für vertragsärztliche Papierdokumente

\begin{tabular}{|l|l|}
\hline $\begin{array}{l}\text { Arbeitsunfähigkeitsbe- } \\
\text { scheinigungen (Teil 1c) }\end{array}$ & 1 Jahr \\
\hline $\begin{array}{l}\text { Betäubungsmittelrezepte } \\
\text { (Durchschriften; verschriebene } \\
\text { Exemplare), Betäubungsmit- } \\
\text { telkartei }\end{array}$ & 3 Jahre \\
\hline $\begin{array}{l}\text { Befunddokumentationsblätter } \\
\text { für Krebsvorsorge, Gesund- } \\
\text { heitsuntersuchung }\end{array}$ & 5 Jahre \\
\hline $\begin{array}{l}\text { Patientenkartei mit allen } \\
\text { Untersuchungsergebnissen } \\
\text { wie Labor, EKG, Sono, eigene } \\
\text { und fremde Arztbriefe }\end{array}$ & 10 Jahre \\
\hline \begin{tabular}{l} 
Erhaltene Überweisungsscheine \\
\hline $\begin{array}{l}\text { Notfall-/Vertreterscheine nach } \\
\text { Muster 19 }\end{array}$
\end{tabular} & 10 Jahr \\
\hline $\begin{array}{l}\text { Sicherungskopien der } \\
\text { Quartalsabrechnung }\end{array}$ & 16 Quartale \\
\hline
\end{tabular}

dere wenn sie steuerlich relevant sein können. Eine 10-Jahres-Frist gilt für Buchungsbelege, Jahresabschlüsse und Inventarlisten. Hier ist der zuständige Steuerberater sicherlich im Einzelfall der richtige Ansprechpartner. In jedem Fall beginnen die Fristen mit dem Ende des Kalenderjahres, in dem das Dokument fertiggestellt wird. Dementsprechend sollten Unterlagen jahrgangsweise archiviert werden, damit sie in gleicher Weise entsorgt werden können. 